\title{
CHARACTERIZING LUMINOSITY EVOLUTION IN THE TEVATRON *
}

\author{
V. Shiltsev, E. McCrory; Fermilab, Batavia, IL, U.S.A..
}

\section{Abstract}

We derive an approximate form of a luminosity evolution in a high intensity hadron collider taking into account the most important phenomena of intrabeam scattering (IBS), beam burn-up due to luminosity and beam-beam effects. It is well known that an exponential decay does not describe luminosity evolution very well unless the lifetime is allowed to vary with time. However, a "1/time" evolution, which this derivation shows is a good approximation, fits data from the Tevatron well.

\section{WEAK-STRONG VS. STRONG-STRONG}

Starting with the well known formula for luminosity in head-on collisions

$$
L=\gamma f_{B} \frac{N_{1} N_{2}}{4 \pi \beta^{*} \varepsilon} H\left(\sigma_{s} / \beta^{*}\right)
$$

and focusing only on the time-dependent factors, then

$$
L(t)=C \frac{N_{1}(t) N_{2}(t)}{\varepsilon(t)} H(t)
$$

( $C$ is a combination of various machine parameters) Then the luminosity lifetime can be expressed as

$$
\tau_{L}^{-1}=\frac{d L(t)}{L(t) d t}=\tau_{N 1}^{-1}+\tau_{N 2}^{-1}-\tau_{\varepsilon}^{-1}+\tau_{H}^{-1}
$$

In equations (1) and (2), the emittance is the average emittance of two round beams $\varepsilon=\left(\varepsilon_{1}+\varepsilon_{2}\right) / 2, H(x)$ is "hourglass factor". In some cases, $L(t)$ can be found analytically, e.g., for e+e- colliders when $N_{1}(t)=N_{2}(t), \varepsilon=$ const and luminosity burn up dominates luminosity lifetime [1]:

$$
L(t)=\frac{L_{0}}{(1+a t)^{2}}
$$

In this section, we consider a more complex example of a hadron collider, where the IBS and beam-beam effects play significant role. If one takes a look into the Tevatron proton-antiproton collider operation in 2004, with range of the initial luminosities between 0.5 to $1.0 \mathrm{e} 32$ $\mathrm{cm}^{-2} \mathrm{~s}^{-1}$, then following observations are valid [2]:

- Proton bunch intensity is much higher than the antiproton, $N_{p}>>N_{a}$

- The biggest contributor into the luminosity decay is growth of beam emittance with a typical time of $\tau_{\varepsilon}$ $\sim 15-20$ hours; the growth is dominated by IBS in the proton beam with small contribution from beam-beam effects;

- Antiproton lifetime of about $\tau_{\mathrm{a}} \sim 20$ to 25 hours by

*Operated by Universities Research Assoc. Inc. under Contract No. DE-AC02-76CH03000 with the US Dept of Energy.
$80 \%$ is determined by the luminosity burn rate and by $20 \%$ by beam-beam effects

- Proton lifetime is mostly driven by beam-beam effects and varies over a wide range: $\tau_{\mathrm{p}} \sim 35$ to 200 hours

- Bunch lengthening due to the IBS (mostly in proton bunches) results in the hourglass factor decay with $\tau_{\mathrm{H}} \sim 70$ to 80 hours

The luminosity lifetime during this period was $\tau_{\mathrm{L}} \sim 7.5$ to 9 hours (averaged over the first two hours of a store).

Thus, the IBS-induced emittance growth and pbar burn up due to luminosity dominate the luminosity decay. For these two effects we can derive analytical formulae for $L(t)$. Theory and simulations of the IBS in the bunches with small longitudinal velocity spread compared to the transverse one predict growth of transverse and longitudinal emittances [3]:

$$
\frac{d \varepsilon_{T}}{d t}=\frac{N_{p} C_{T}}{\varepsilon_{T}{ }^{1.5} \varepsilon_{L}^{0.5}} \text { and } \frac{d \varepsilon_{L}}{d t}=\frac{N_{p} C_{L}}{\varepsilon_{T}{ }^{1.5} \varepsilon_{L}^{0.5}}
$$

$C_{L}$ and $C_{T}$ are constants determined by machine parameters. From (5) one gets an asymptotic of $\varepsilon_{\mathrm{T}} \propto \varepsilon_{\mathrm{L}} \propto$ $t^{1 / 3}$ if $t \rightarrow \infty$, and, approximately:

$$
\varepsilon_{T}(t) \approx \varepsilon_{T 0}\left(1+\frac{t}{\tau_{T} \beta}\right)^{\beta} \text { and }
$$

$$
\varepsilon_{L}(t) \approx \varepsilon_{L 0}\left(1+\frac{t}{\tau_{L} \beta}\right)^{\beta} \text { where } \beta \approx 1 / 3
$$

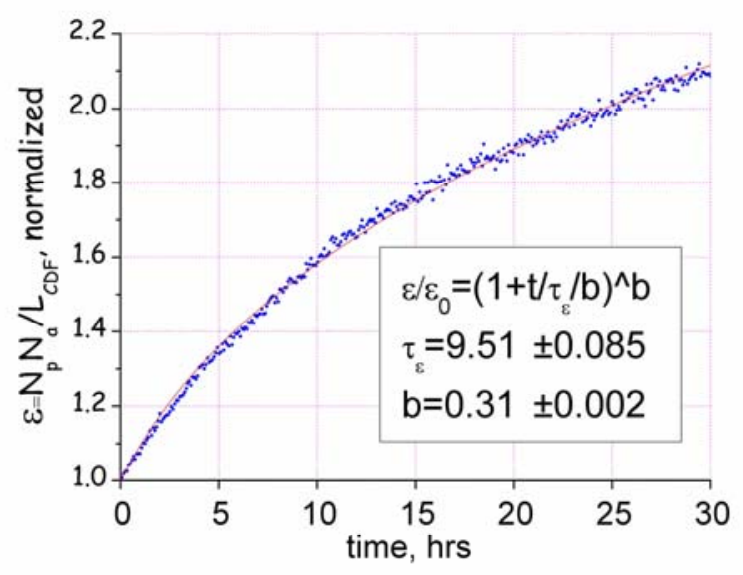

Fig.1: $\varepsilon_{\text {eff }}$ vs. time in store \#3655; points are the data from the store and the line is a fit to the data.

Given $d \varepsilon_{y} / d t \approx 0.5 d \varepsilon_{x} / d t$ for protons and both transverse and longitudinal emittance growth rates are smaller for antiprotons: $d \varepsilon_{a} / d t<<d \varepsilon_{p} / d t$; c) $\tau_{\mathrm{H}}$ $>>\tau_{\varepsilon}$ one gets: 


$$
\varepsilon_{\text {eff }}(t)=C \frac{N_{p} N_{a}}{L}=\frac{\varepsilon_{p}+\varepsilon_{a}}{2 H} \approx \varepsilon_{0}\left(1+\frac{t}{\tau_{\varepsilon} \alpha}\right)^{\alpha}
$$

where $\alpha \approx 1 / 3 \quad$ (7).

Fig.1 demonstrates that such an approximation (7) satisfactorily describes emittance evolution in the Tevatron store $3655(07 / 14 / 2004)$.

The beam burn up rate due to luminosity is

$$
\frac{d N_{a}}{N_{a} d t}=-\frac{\sigma_{i n} L}{N_{a}}=\frac{\text { const }}{\varepsilon_{\text {eff }} / N_{p}}
$$

and, given that $\tau_{N p}>>\tau_{N a}$, one gets

$$
\frac{-d N_{a}}{N_{a} d t}=\frac{C_{a}}{\left(1+t / \tau_{a} / \omega\right)^{\omega}}
$$

where $\omega \approx \alpha \approx 1 / 3$ and $\tau_{a} \approx \tau_{\varepsilon}$ (9).

Solution of Eq.(9) is

$$
\frac{N_{a}}{N_{0}}=\exp \left(-\frac{\omega \tau_{a} C_{a}}{1-\omega}\left[\left(1+t / \tau_{a} / \omega\right)^{1-\omega}-1\right]\right)
$$

(10),

and combining $\operatorname{Eqs}(7)$ and (10), one has

$$
\frac{L}{L_{0}}=\frac{\exp \left(-\frac{\omega \tau_{a} C_{a}}{1-\omega}\left[\left(1+t / \tau_{a} / \omega\right)^{1-\omega}-1\right]\right)}{(1+t / \tau / \omega)^{\omega}}
$$

Eq.(11) gives approximate evolution of luminosity in the case when antiproton beam intensity is much smaller than the proton beam one.

For the "strong-strong" case, when intensities of both beams are comparable $N_{1}(t) \approx N_{2}(t)=N(t)$, (situation anticipated later in the Tevatron Run II, when after various upgrades antiproton bunch intensity will reach half of the proton one), Eq. (7) is still valid, and if we introduce normalized intensity $F=N(t) / N(t=0)$, then luminosity evolution is given by

$$
\frac{L}{L_{0}}=\frac{F^{2}}{\left(1+t / \tau_{\varepsilon} / \alpha\right)^{\alpha}}
$$

and, therefore,

$$
\frac{d F}{d t}=-\frac{\sigma_{i n} L}{N_{0}}=-\frac{C_{0} F^{2}}{\left(1+t / \tau_{\varepsilon} / \alpha\right)^{\alpha}}
$$

Solutions of Eqs.(12) and (13) are:

$$
\begin{gathered}
F=\frac{N}{N_{0}}=\frac{1}{1+A\left[\left(1+t / \tau_{\varepsilon} / \alpha\right)^{1-\alpha}-1\right]} ; \\
A=C_{0} \tau_{\varepsilon} \alpha /(1-\alpha) \\
L=\frac{L_{0}}{\left[1+A\left[\left(1+t / \tau_{\varepsilon} / \alpha\right)^{1-\alpha}-1\right]\right]^{2}\left(1+t / \tau_{\varepsilon} / \alpha\right)^{\alpha}}
\end{gathered}
$$

These equations can be further approximated by formulae having correct asymptotics at $t>\tau_{\varepsilon}$ :

$$
\begin{aligned}
& N \approx \frac{N_{0}}{\left[1+t / \tau_{N} / \alpha\right]^{\alpha}} ; \quad L \approx \frac{L_{0}}{\left[1+t / \tau_{L} / \mu\right]^{\mu}} ; \\
& \alpha \approx 1 / 3 ; \mu \approx 2-\alpha=5 / 3
\end{aligned}
$$

Interestingly, such simple form of approximation as in Eq.(15) - rational of fractional power law - satisfactorily works for the "weak-strong" case considered above in Eqs. $(10,11)$ when the power coefficient is reduced $\mu<5 / 3$, see Fig.2.

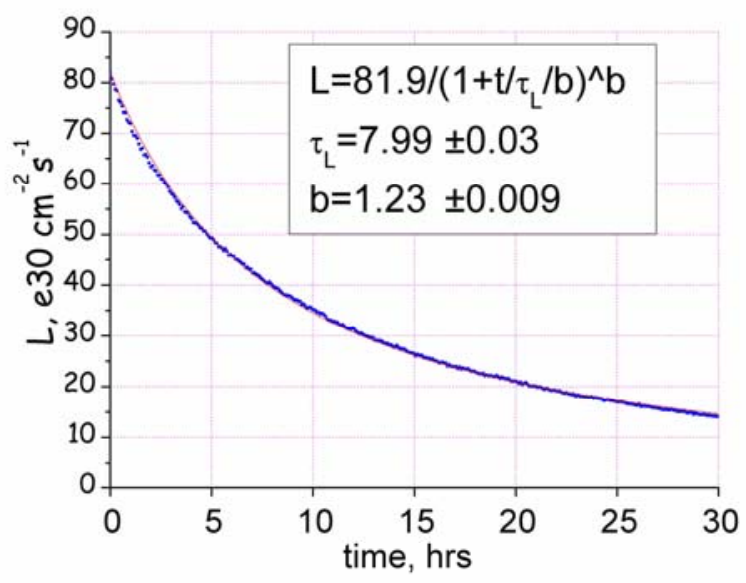

Fig.2: L vs time in store \#3655; points are measured data from this store, the line is a fit to the data.

These results are applied in our companion paper [4].

\section{CONCLUSION}

An approximate form for the luminosity evolution in a hadron collider is derived. This analysis takes into account IBS in its various, well-known forms: beam burnup due to luminosity and beam-beam effects. A "1/time" evolution, which this derivation shows is a good approximation, fits data from the Tevatron well.

\section{REFERENCES}

[1] Handbook of Accelerator Physics and Engineering, A. W. Chao, M. Tigner, eds., World Scientific, (1998), p.217.

[2] V. Shiltsev, beams-doc-1357, see also in Proc. EPAC 2004 (Lucern).

[3] D. Finley, FNAL-TM-1646 (1989); V. Lebedev, in Proc. IEEE PAC 2003 (Portland).

[4] E. McCrory, et al., "Fitting the luminosity decay in the Tevatron," paper \#2503 (TPAP036), this conference. 